\title{
A INFLUÊNCIA DO TREINAMENTO DE VENDEDORES NA SATISFAÇÃO DOS CLIENTES DE UMA REDE DE CONCESSIONÁRIAS DE VEÍCULOS, POR MEIO DA MODELAGEM DE EQUAÇÕES ESTRUTURAIS
}

\author{
THE INFLUENCE OF SALESPEOPLE TRAINING ON THE \\ CUSTOMER SATISFACTION OF A NETWORK'S VEHICLE \\ DEALERS BY STRUCTURAL EQUATIONS MODELING
}

\section{LA INFLUENCIA DE LOS ENTRENAMIENTOS DE VENDEDORES EN LA SATISFACCIÓN DE LOS CLIENTES DE UNA RED DE CONCESIONARIOS DE VEHÍCULOS POR MEDIO DEL MODELADO DE ECUACIONES ESTRUCTURALES}

Fábio Hiroshi Tomoyose

Mestre em Administração pela Universidade Municipal de São Caetano do Sul

hiroshi_fabio@hotmail.com

\author{
Ana Cristina de Faria \\ Pós-Doutora em Gestão de Operações e Logística pela \\ Fundação Getúlio Vargas (FGV), Brasil / Doutora e \\ Mestre em Controladoria e Contabilidade pela \\ Universidade de São Paulo (USP), Brasil / Consultora e \\ Perita Contábil \\ anacfaria@uol.com.br
}

\section{Marcos Antonio Gaspar \\ Doutor e Mestre em Administração pela Universidade de São Paulo (USP), Brasil / Professor do Programa de Pós-Graduação em Informática e Gestão do \\ Conhecimento da Universidade Nove de Julho \\ (UNINOVE), Brasil \\ marcos.antonio@uninove.br}

\section{Edson Keyso de Miranda Kubo}

Doutor em Administração pela Fundação Getúlio Vargas (FGV), Brasil / Mestre em Administração pela Kobe University, Japão, e Universidade Estadual Paulista (UNESP), Brasil / Professor do Programa de Pós-Graduação em Administração da Universidade Municipal de São Caetano do Sul (USCS) edsonkubo@uscs.edu.br
Contextus

ISSNe 2178-9258

Organização: Comitê Científico Interinstitucional Editor Científico: Carlos Adriano Santos Gomes Avaliação: double blind review pelo SEER/OJS

Recebido em 11/03/2017 Aceito em 08/12/2017 $2^{a}$ versão aceita em 26/12/2017

\footnotetext{
RESUMO

Este estudo teve como objetivo constatar a influência do treinamento de vendedores das concessionárias de veículos do estado do Rio de Janeiro na satisfação dos clientes de uma montadora. Adotou-se o tratamento de dados secundários e a Modelagem de Equações Estruturais, com estimação dos mínimos quadrados parciais, em uma amostra de 130 concessionárias, 936 respondentes à pesquisa de satisfação e a análise do desempenho nos treinamentos de 1.158 vendedores. Concluiu-se que os treinamentos realizados pelos vendedores das concessionárias exercem função estratégica para a criação de valor ao cliente e geração de vantagem competitiva, mesmo não possuindo relação direta com a satisfação dos clientes. Mediante os resultados
} 
apresentados, pode-se afirmar que os treinamentos realizados pelos vendedores estão ligados diretamente às percepções dos clientes acerca da qualidade em serviços oferecida pela montadora e sua rede concessionária.

Palavras-chave: Indústria Automobilística. Modelagem de Equações Estruturais. Qualidade Percebida em Serviços. Satisfação do Cliente. Treinamento de Vendedores.

\begin{abstract}
This study aimed to verify the influence of salespeople training of vehicle dealers in Rio de Janeiro state on the customers satisfaction of an automaker. We adopted the secondary data treatment and the Structural Equation Modeling, with partial least squares estimation, in a sample of 130 dealers, 936 respondents to the satisfaction survey and the analysis of training performance of 1,158 vendors. It was concluded that the salespeople training of vehicle dealers plays a strategic role in creating value to the customer and generating competitive advantage, even though it has no direct relation with the customer satisfaction. Through the presented results, it can be affirmed that the trainings carried out by the vendors are directly linked to the perceptions of the clients about the quality in services offered by the automaker and its concessionaire network. By the presented results, it can be said that salespeople trainings are directly linked to the customer perceptions about service quality offered by the automaker and its dealers network.
\end{abstract}

Keywords: Automotive industry. Customer satisfaction. Salespeople training. Service perceived quality. Structural Equations Modeling.

\title{
RESUMEN
}

Este estudio tuvo como objetivo constatar la influencia del entrenamiento de vendedores de las concesionarias de vehículos del estado de Río de Janeiro en la satisfacción de los clientes de un fabricante de vehículos. Se adoptó el tratamiento de datos secundarios y la técnica de Modelado de Ecuaciones Estructurales, con la estimación de los mínimos cuadrados parciales, en una muestra de 130 concesionarias, 936 respondedores a la encuesta de satisfacción y el análisis del desempeño en los entrenamientos de 1.158 vendedores. Se concluyó que los entrenamientos realizados por los vendedores de las concesionarias asumen función estratégica para la creación de valor al cliente y la generación de ventaja competitiva, aun no teniendo relación directa con la satisfacción de los clientes. Por medio de los resultados presentados, se puede afirmar que los entrenamientos de vendedores están ligados directamente a las percepciones de los clientes acerca de la calidad en servicios, ofrecida por el fabricante de vehículos y su red concesionarias.

Palabras clave: Satisfacción del cliente. Entrenamiento de vendedores. Calidad percibida en servicios. Industria Automovilística. Modelados de ecuaciones estructurales.

\section{INTRODUÇÃO}

A indústria automobilística brasileira tem se mostrado cada vez mais relevante no cenário internacional, seja pela sua capacidade exportadora em momentos de crise ou pelo público de consumidores potenciais que apresenta no mercado interno. Dados da Associação Nacional dos Fabricantes de Veículos Automotores - ANFAVEA (2017) mostram acréscimo de 53,5\% no volume de exportações de automóveis e comerciais leves de 2014 a novembro de 2017.
De acordo com a Federação Brasileira dos Distribuidores de Veículos Automotores - FENABRAVE (2017), o Brasil, em 2014, manteve a quarta posição no ranking dos países que mais comercializaram automóveis e comerciais leves no mundo, com 3.328.958 unidades, atrás somente da China, EUA e Japão. Apesar da recessão econômica instaurada no Brasil desde 2014 (BARBOSA FILHO, 2017), o país ainda tem atraído novas montadoras, cujos objetivos voltam-se à conquista do mercado brasileiro automobilístico (STURGEON; 
BIESEBROECK; $\quad$ GEREFFI， 2008; ANFAVEA, 2017).

Diante desse contexto, a satisfação do cliente é imperativa para aqueles que desejam manter-se ou tornar-se competitivos, uma vez que clientes insatisfeitos migram para a concorrência e criam expectativas negativas em outros potenciais clientes (FORNELL, 2007). Assim, uma das estratégias adotadas pelas montadoras para a satisfação dos seus clientes, no tocante à qualidade dos serviços prestados, refere-se ao desenvolvimento dos colaboradores da linha de frente de sua rede de concessionárias, notadamente por meio de treinamentos (RAPHAËLE; RAFER, 1998).

O treinamento de colaboradores, conforme defendido por Salas e CannonBowers (2001), dotando-os de maior autonomia e preparo para garantir a qualidade no atendimento, pode implicar maior comprometimento no trabalho e na satisfação dos clientes (JAMES; JONES, 2014). Colaboradores capacitados podem influenciar as decisões de compra, as percepções e as expectativas dos clientes, além de representarem investimentos significativos que impactam nos custos das montadoras (COSTA et al., 2013).

Nesse sentido, esta pesquisa parte do seguinte questionamento: em que medida a satisfação dos clientes de uma rede de concessionárias de automóveis do estado do Rio Janeiro é influenciada pelo treinamento dos vendedores da referida rede? Para se responder a questão proposta, estabeleceu-se como objetivo: constatar a influência do treinamento em vendas das concessionárias do Rio de Janeiro na satisfação dos clientes de uma montadora.

O tema do treinamento de vendedores e a sua influência na satisfação dos clientes, adotando-se a abordagem quantitativa e tendo como foco o setor automobilístico, ainda é pouco explorada pela literatura especializada, apesar da relevância multidisciplinar que envolve aspectos relacionados ao planejamento estratégico, gestão de pessoas, gestão de custos e finanças. Nesse sentido, faz-se necessário explorar o tema e delinear um método capaz de estimar e avaliar os programas de treinamento, bem como verificar a relação existente entre tais programas e os resultados organizacionais esperados.

\section{REFERENCIAL TEÓRICO}

\subsection{Satisfação do cliente}

Abordada na literatura especializada de marketing e comportamento do consumidor, bem como 
relacionada a diferentes aspectos no contexto acadêmico e empresarial, a satisfação do cliente assume múltiplas facetas, que partem da análise comportamental e das percepções dos clientes, à sua relação com outras variáveis (FORNELL et al., 1996; CRONIN; BRADY; HULT, 2000; SILVA; BERTRAND，2008; STAVRINOUDIS; PSIMOULIS, 2017).

De acordo com Oliver (1980), a satisfação do cliente é um estado emocional resultante da sua experiência de consumo, nas quais os esforços empreendidos pela organização na oferta de um produto ou serviço superaram as suas expectativas. No contexto organizacional, a satisfação dos clientes representa o foco de objetivos e estratégias, pois clientes satisfeitos tornam-se leais e, consequentemente, eleva-se a probabilidade de recompra (ANDERSON; SULLIVAN, 1993; ANDERSON; FORNELL; LEHMMANN, 1994; ANDERSON; FORNELL; RUST, 1997; SILVA， 2006; FORNELL， 2007; WILLIANS; NAUMANN, 2011; ELLINGER et al., 2012; SPARROW; OTAYE-EBEDE, 2014).

A relevância do tema da satisfação do cliente pode ser constatada pelo Índice Americano de Satisfação do Cliente (ACSI), que representa nos EUA um indicador econômico e do nível de competitividade entre empresas, setores e até mesmo países (FORNELL et al., 1996; URDAN; RODRIGUES, 1999; MOURA; GONÇALVES, 2005; FORNELL, 2007).

A literatura aborda dimensões distintas, porém complementares, que avaliam as percepções dos clientes, uma sob a ótica da satisfação do cliente e outra sob a ótica da qualidade percebida em serviços.

\subsection{Qualidade percebida em serviços}

A qualidade percebida em serviços pode ser entendida como o julgamento dos clientes sobre a superioridade ou excelência de uma organização, baseado nos atributos de um determinado serviço avaliado pelo usuário (PARASURAMAN; ZEITHAML; BERRY, 1988). Trata-se do resultado ou desempenho global das organizações na oferta de serviços ao seu público consumidor

(ZEITHAML; BITNER; GREMLER, 2011).

Conforme explicam Cronin e Taylor (1992); Cronin, Brady e Hult (2000), Ros e Benavent (2002), a qualidade percebida em serviços é um fator predominante e isolado da percepção do cliente, ou seja, é tida como uma entidade única que pode ser estimada com base em cinco outros fatores ou dimensões, 
conforme definidos por Parasuraman, Zeithaml e Berry (1988):

1) Tangíveis: referem-se às evidências físicas;

2) Confiança: representa o comprometimento da empresa ou do colaborador;

3) Competência: envolve as habilidades e conhecimentos da empresa ou dos colaboradores;

4) Segurança: conhecimento e cortesia dos colaboradores; e

5) Empatia: compreensão e identificação com o cliente.

Para Waligóra e Waligóra (2007) e Rahman, Khan e Haque (2012), a qualidade percebida pelo cliente, seja em produtos ou serviços, pode ser compreendida como a determinante da sua satisfação. Na visão dos autores, há uma relação direta e positiva entre a qualidade percebida em serviços e a satisfação dos clientes.

\subsection{Qualidade percebida em serviços vs. satisfação dos clientes}

Apesar de apresentarem algumas semelhanças, os conceitos de qualidade percebida em serviços e de satisfação do cliente são essencialmente diferentes quanto às suas causas e seus respectivos efeitos (ZEITHAML;
GREMLER, 2011). A qualidade em serviços reflete o desempenho global de uma organização na oferta de serviços, ao passo que o conceito da satisfação do cliente é mais amplo e considera, além da experiência com a aquisição de um serviço ou produto, fatores intrínsecos a cada indivíduo, tais como: personalidade, influência familiar e cultural (ZEITHAML; BITNER; GREMLER, 2011).

A literatura apresenta inúmeras pesquisas que estabelecem relações entre a qualidade percebida em serviços e a satisfação do cliente, bem como as relações destas variáveis com outras variáveis de interesse de pesquisa, conforme estudos realizados, por:

a) Silva e Bertrand (2008) que estabeleceram uma relação entre a previsão de vendas e a satisfação do cliente;

b) Vieira, Matos e Slongo (2009) que avaliaram as relações entre qualidade de serviço, satisfação, valor percebido e lealdade do cliente;

c) Willians e Naumann (2011) que estudaram a relação entre a satisfação do cliente, lealdade e desempenho econômico-financeiro;

d) Rahman, Khan e Haque (2012) que pesquisaram as relações entre a qualidade percebida em serviços, satisfação dos clientes, qualidade técnica e 
funcional, imagem corporativa, preço percebido, influências internas e externas; $\mathrm{e}$

e) Lien, Cao e Zhou (2017) que analisaram as relações entre a qualidade em serviços, satisfação, motivação de permanência e intenções de uso, entre outras pesquisas.

Face aos argumentos presentes na literatura supracitada, a hipótese inicial inferida, com o objetivo de buscar respostas ao problema proposto na pesquisa, é:

H1. A qualidade percebida pelos clientes, acerca dos serviços prestados pelos vendedores das concessionárias, influencia direta e positivamente a sua satisfação.

Parte-se do pressuposto de que a qualidade percebida em serviços e a satisfação do cliente representam dois construtos distintos, sendo o primeiro determinante do segundo, conforme afirmam Cronin, Brady e Hult (2000), Waligóra e Waligóra (2007), Rahman, Khan e Haque (2012), Lien, Cao e Zhou (2017). O presente estudo procurou analisar a conexão entre os construtos mencionados e o treinamento dos vendedores de automóveis.

\subsection{Treinamento de vendedores}

Os treinamentos proporcionados por uma montadora de veículos à sua rede de concessionárias são, na visão de Raphaële e Rafer (1998), um elemento estratégico para o crescimento e ganho de vantagem competitiva. Para esses autores, as montadoras empenhadas na capacitação da sua rede de concessionárias visam à qualidade dos serviços prestados, padronização, à atualização e ao desenvolvimento dos colaboradores da rede, para que resultem na satisfação dos seus clientes.

Mais importante do que o monitoramento da montadora por meio de instrumentos capazes de mensurar a satisfação dos clientes, é o comportamento das pessoas envolvidas no contato direto com os clientes, tais como: vendedores, atendentes, recepcionistas, operadores e todos aqueles que representam a interface entre a organização e os clientes (DENTON, 1990).

Rockenbach et al. (2013) afirmam que a capacitação de pessoas, face à atual conjuntura da gestão de pessoas, que visa o alinhamento da estratégia organizacional com o planejamento, representa um fator de vantagem competitiva à organização. Ainda para os autores, o termo capacitação é tratado de forma genérica e engloba as 
concepções de ensino, desenvolvimento, educação e treinamento. Já Sparrow e Otaye-Ebede (2014) enfatizam o papel da gestão de pessoas no provimento de capital intelectual e capacitação que implique em melhoria da qualidade em serviços e incremento da competitividade da empresa.

$$
\text { Para Costa et al. (2013), }
$$

Stavrinoudis e Psimoulis (2017), o treinamento é capaz de potencializar o desempenho dos funcionários e contribuir para a satisfação dos clientes, bem como para os resultados financeiros de uma organização. Para os autores, o treinamento dos funcionários, entre outros benefícios, reside no desenvolvimento profissional e pessoal que são elementos fundamentais da motivação. A prática de treinamentos representa um fator de vantagem competitiva e destaque em relação à concorrência, dada a sua relação com a satisfação dos clientes (COSTA et al., 2013).

No entanto, o estudo de Hernández et al. (2011) realizado com 43 funcionários e 191 clientes em hotéis no México, revelou a existência de uma relação na capacitação dos funcionários e a qualidade percebida em serviços pelos clientes, e não uma relação com satisfação destes. Segundo os autores, não há uma relação significativa entre os treinamentos realizados pelos funcionários e a satisfação dos clientes, e sim uma relação direta e positiva com a qualidade percebida em serviços.

Para Benossi (2009), um dos principais problemas enfrentados pela administração é a difícil mensuração dos resultados provenientes dos esforços envolvidos na capacitação profissional em relação aos investimentos realizados. Para a autora, as dificuldades em mensurar os resultados advindos da capacitação, podem gerar no administrador um sentimento de incerteza, pois não há exatidão que estabeleça critérios para o direcionamento de recursos financeiros, nem tampouco exatidão que justifique os resultados obtidos (BENOSSI, 2009).

Conforme explicam Thirkell e Ashman (2014), Benossi (2009), Tasca, Ensslin e Ensslin (2012), Costa et al. (2013), Stavrinoudis e Psimoulis (2017), todo o processo de treinamento necessita de avaliação e monitoramento, de modo que possam ser analisados os benefícios advindos, verificando se os objetivos préestabelecidos foram atingidos e possibilite o melhoramento, ajuste ou até mesmo o cancelamento de treinamentos que não estejam alinhados com os resultados esperados.

A avaliação e monitoramento do processo de treinamento, além de proporcionar uma visão do crescimento 
individual de cada colaborador, devem proporcionar uma visão do crescimento organizacional, ou seja, uma visão das contribuições provenientes dos treinamentos que justifiquem os investimentos e tudo aquilo que as organizações esperam com esta prática (BENOSSI, 2009; HERNÁNDEZ et al., 2011; STAVRINOUDIS; PSIMOULIS, 2017).

Neste sentido, faz-se necessária a apropriação de um instrumento capaz de mensurar e avaliar os programas de treinamento, bem como verificar a relação existente entre estes programas e os resultados organizacionais esperados. Levando-se em consideração o objeto (montadora e rede de concessionárias) e o objetivo (a influência do treinamento na satisfação dos clientes) do presente estudo, bem como o pressuposto de que o foco das estratégias organizacionais deve ser a satisfação dos clientes e sua consequente lealdade (ANDERSON; FORNELL; LEHMMANN, 1994; FORNELL et al., 1996; ANDERSON; FORNELL; RUST, 1997; ANDREASSEN; LINDESTAD, 1998; RAPHAËLE; RAFER; 1998; FORNELL 2007; WILLIANS; NAUMANN, 2011; ELLINGER et al., 2012), inferiu-se uma segunda hipótese:

H2. Os treinamentos realizados pelos vendedores das concessionárias influenciam direta e positivamente a satisfação dos clientes.

Não obstante, levando-se em consideração o entendimento de que os construtos da qualidade percebida em serviços e da satisfação dos clientes são essencialmente diferentes quanto as suas causas e os efeitos resultantes, bem como o estudo de Hernández et al. (2011) que constatou uma relação direta e positiva dos treinamentos com a qualidade percebida em serviço, em vez de uma relação direta como a satisfação dos clientes, inferiu-se uma terceira hipótese:

H3. Os treinamentos realizados pelos vendedores das concessionárias influenciam direta e positivamente a qualidade percebida pelos clientes, acerca dos serviços prestados.

Com a inferência da terceira e última hipótese, foi possível estabelecer as relações entre o treinamento dos vendedores, a qualidade percebida em serviços pelos clientes e a sua satisfação, mediante o delineamento de um modelo conceitual.

\subsection{Modelo conceitual}

A análise a ser desenvolvida parte dos pressupostos iniciais levantados na literatura especializada acerca da satisfação dos clientes, qualidade percebida em 
serviços e o treinamento dos vendedores, bem como das relações estabelecidas entre esses construtos, mediante a inferência de hipóteses. De acordo com Gil (2002, p. 43), "para confrontar a visão teórica com os dados da realidade, torna-se necessário traçar um modelo conceitual e operativo da pesquisa".

Quadro 1 - Síntese dos conceitos tratados na pesquisa
Nesse sentido, o Quadro 1 a seguir, apresenta a síntese dos pressupostos tratados anteriormente, mediante os conceitos, definições e os respectivos autores:

\begin{tabular}{|c|c|c|}
\hline Con & Definições & Autores \\
\hline $\begin{array}{l}\text { Satisfação dos } \\
\text { Clientes } \\
\text { (SC) }\end{array}$ & $\begin{array}{l}\text { Trata-se do resultado da experiência de consumo } \\
\text { dos clientes e objeto das estratégias } \\
\text { organizacionais, possuindo como determinante a } \\
\text { qualidade percebida em serviços (QS) e como } \\
\text { consequência a lealdade dos clientes. }\end{array}$ & $\begin{array}{c}\text { Oliver (1980); Anderson e Sullivan } \\
\text { (1993); Anderson, Fornell e Lehmmann } \\
\text { (1994); Anderson, Fornell e Rust (1997); } \\
\text { Silva (2006); Fornell (2007); Willians e } \\
\text { Naumann, (2011); Ellinger et al. (2012); } \\
\text { Sparrow e Otaye-Ebede (2014); Lien, Cao } \\
\text { e Zhou (2017); Stavrinoudis e Psimoulis } \\
\text { (2017) }\end{array}$ \\
\hline $\begin{array}{l}\text { Qualidade } \\
\text { Percebida em } \\
\text { Serviços } \\
\text { (QS) }\end{array}$ & $\begin{array}{c}\text { Refere-se ao desempenho global da organização } \\
\text { na prestação de serviços, segundo a avaliação } \\
\text { dos clientes acerca dos tangíveis, confiança, } \\
\text { segurança, empatia e competência. Possui } \\
\text { relação direta e positiva com a satisfação dos } \\
\text { clientes (SC). }\end{array}$ & $\begin{array}{l}\text { Parasuraman, Zeithaml e Berry (1988); } \\
\text { Zeithaml, Bitner e Gremler (2011); } \\
\text { Cronin e Taylor (1992); Cronin, Brady e } \\
\text { Hult (2000); Ros e Benavent (2002); } \\
\text { Waligóra e Waligóra (2007); Rahman, } \\
\text { Khan e Haque (2012); Lien, Cao e Zhou } \\
\text { (2017) }\end{array}$ \\
\hline $\begin{array}{l}\text { Treinamento de } \\
\text { Vendedores } \\
\text { (TR) }\end{array}$ & $\begin{array}{l}\text { Elemento estratégico de vantagem competitiva, } \\
\text { capaz de potencializar as vendas, motivar } \\
\text { colaboradores e incrementar o resultado geral das } \\
\text { organizações. Trata-se de um construto de difícil } \\
\text { estimação e que pode estar relacionado tanto com } \\
\text { a satisfação dos clientes (SC), quanto com a } \\
\text { qualidade em serviços por eles percebida (QS). }\end{array}$ & $\begin{array}{l}\text { Raphaële e Rafer (1998); Benossi (2009); } \\
\text { Hernández et al. (2011); Tasca, Ensslin e } \\
\text { Ensslin (2012); Costa } \text { et al. (2013); } \\
\text { Rockenbach } \text { et al. (2013); Costa } \text { et al. } \\
\text { (2013); Sparrow e Otaye-Ebede (2014); } \\
\text { Thirkell e Ashman (2014); Stavrinoudis e } \\
\text { Psimoulis (2017) }\end{array}$ \\
\hline
\end{tabular}

Fonte: elaboração dos autores.

O Quadro 1 mostra a síntese dos construtos tratados no referencial teórico, mediante a indicação de cada conceito, as respectivas definições e autores que os defendem. A partir do referido quadro foi possível abstrair e propor o modelo conceitual da pesquisa, presente na Figura $1:$ 
Figura 1 - Modelo conceitual proposto

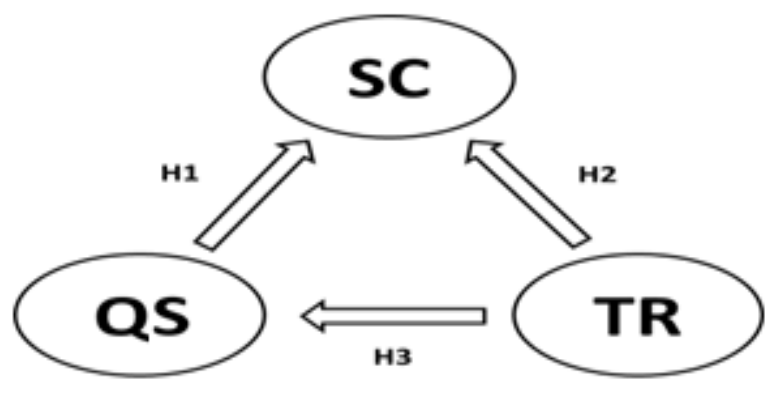

Fonte: elaboração dos autores.

A Figura 1 apresenta cada elipse como uma variável latente, não observada, quais sejam: qualidade percebida em serviços (QS); treinamento de vendedores (TR) e satisfação do cliente (SC). As setas indicam a relação causal entre as variáveis latentes ou construtos, e as letras ' $\mathrm{H}$ ' indicam as hipóteses inferidas e já destacadas na plataforma teórica deste estudo.

Com o modelo conceitual proposto - baseado na questão que se pretendeu responder, nos pressupostos encontrados na literatura especializada acerca e nas hipóteses levantadas -, recorreu-se à metodologia científica que norteou a presente pesquisa. Cabe ressaltar que, deste ponto em diante, os construtos, objetos do presente estudo, foram tratados por suas siglas, quais sejam: QS - Qualidade Percebida em Serviços, TR - Treinamento de Vendedores e SC - Satisfação dos Clientes, de modo a facilitar a análise dos resultados e evitar o excesso das mesmas expressões.

\section{METODOLOGIA PESQUISA}

DA

Trata-se de um estudo exploratório e descritivo, com abordagem quantitativa (GIL, 2002; MALHOTRA, 2006). Partiuse da análise de uma pesquisa de satisfação, adotada por uma montadora do setor automobilístico no mês de julho de 2013 e de relatórios de aproveitamento em treinamentos da rede pertencente à marca (concessionárias), cujas datas retroagem dois meses à data de aplicação da pesquisa de satisfação. Atendendo ao pedido da montadora, não foram revelados os nomes das concessionárias, nem a marca que representam, sendo nominados neste estudo somente por concessionárias e montadora.

A população da pesquisa envolveu 100\% das 130 concessionárias da marca atuantes no estado do Rio de Janeiro, tendo alcançado ainda $7 \%$ dos clientes das concessionárias respondentes à pesquisa de satisfação e $100 \%$ de seus vendedores para 
avaliação do desempenho nos treinamentos. Assim, as amostras obtiveram o total de 936 respondentes à pesquisa de satisfação e a análise do desempenho nos treinamentos de 1.158 vendedores.

Em paralelo, foram conduzidas entrevistas não estruturadas com os responsáveis das áreas de treinamento e marketing da montadora, que contribuíram para a constatação da relevância do tema abordado neste estudo.

\subsection{Análise do modelo de pesquisa de satisfação adotado pela montadora}

A pesquisa de satisfação da montadora, objeto de análise desta pesquisa, consiste em uma survey, aplicada via telefone, na forma de um questionário estruturado composto por 16 perguntas fechadas, cujas respostas foram computadas em uma escala ordinal do tipo Likert, de 1 a 5, na qual 1 significava 'totalmente insatisfeito' e 5 'totalmente satisfeito' (GIL, 2002; HAIR Jr. et al., 2005). A medida padrão adotada pela montadora estabelecia que ao menos $7 \%$ dos clientes adquirentes dos veículos zero quilômetro fossem entrevistados.

Constatou-se, também, que a seleção dos clientes para aplicação das entrevistas era realizada aleatoriamente por intermédio de um sistema, sem quaisquer restrições do tipo de veículo adquirido, sexo ou idade. É importante destacar que a pesquisa de satisfação de que tratou este estudo, refere-se à satisfação dos clientes com os serviços de vendas de veículos zero quilômetro. Os resultados de cada entrevista foram registrados em um banco de dados, tendo sido acessados e controlados pelas notas geradas em cada questão.

As notas geradas para cada concessionária podiam ser visualizadas ou obtidas por meio de relatórios, cujos resultados eram dispostos e interpretados em uma escala de 0 a 100 , por meio da relação: número de respondentes que avaliaram um determinado item do questionário com a nota cinco, pelo número total de respondentes, multiplicado por 100. A análise inicial possibilitou o desenvolvimento do Quadro 2, a seguir, e serviu de base para a construção do modelo de mensuração adotado na presente pesquisa: 
Quadro 2 - Relação das variáveis observadas (indicadores) por variável latente.

\begin{tabular}{|c|c|}
\hline Indicadores & Descrição \\
\hline \multicolumn{2}{|r|}{ Indicadores reflexivos da variável latente endógena da satisfação dos clientes (SC) } \\
\hline $1 \mathrm{SC}$ & Indicador da satisfação geral do cliente. \\
\hline $2 \mathrm{SC}$ & Indicador da satisfação do cliente com o desempenho da concessionária na venda do veículo. \\
\hline $3 \mathrm{SC}$ & Indicador da satisfação do cliente com o tempo despendido em todo o processo de compra. \\
\hline 4SC & Indicador da satisfação do cliente dada as suas expectativas. \\
\hline \multicolumn{2}{|c|}{ Indicadores reflexivos da variável latente exógena e endógena da qualidade percebida em serviços (QS) } \\
\hline 1QS & Avaliação do cliente acerca da infraestrutura da concessionária. \\
\hline 2QS & Avaliação do cliente acerca do atendimento prestado pelo vendedor. \\
\hline $3 \mathrm{QS}$ & Avaliação do cliente acerca da educação e atenção despendida pelo vendedor. \\
\hline 4QS & $\begin{array}{l}\text { Avaliação do cliente acerca da prestatividade do vendedor na identificação do veículo de } \\
\text { acordo com as suas necessidades. }\end{array}$ \\
\hline $5 \mathrm{QS}$ & $\begin{array}{l}\text { Avaliação do cliente acerca da desenvoltura do vendedor na explicação das especificações do } \\
\text { veículo. }\end{array}$ \\
\hline 6QS & $\begin{array}{l}\text { Avaliação do cliente acerca da oferta de teste com o veículo rodando, pelo vendedor (test } \\
\text { drive). }\end{array}$ \\
\hline 7QS & $\begin{array}{l}\text { Avaliação do cliente acerca dos procedimentos adotados pela concessionária para liberação do } \\
\text { veículo. }\end{array}$ \\
\hline $8 Q S$ & $\begin{array}{l}\text { Avaliação do cliente acerca do tempo estabelecido pela concessionária para a liberação do } \\
\text { veículo. }\end{array}$ \\
\hline 9QS & Avaliação do cliente acerca do estado do veículo no ato da entrega das chaves. \\
\hline 10QS & Avaliação do cliente acerca das especificações do veículo, dadas no ato da entrega. \\
\hline $11 \mathrm{QS}$ & Avaliação do cliente acerca da explicação sobre \\
\hline $12 \mathrm{QS}$ & Avaliação do cliente acerca da pesquisa de satisfação adotada pela montadora. \\
\hline \multicolumn{2}{|r|}{ Indicadores formativos da variável latente exógena do treinamento de vendedores (TR) } \\
\hline 1TR & $\begin{array}{l}\text { Indicador de desempenho dos vendedores em treinamentos de 'atendimento nas vendas', por } \\
\text { concessionária - (média das notas dos cursos de atendimento por concessionária + avaliação do } \\
\text { cliente } 2 \mathrm{QS}) / 2 \text {. }\end{array}$ \\
\hline 2TR & $\begin{array}{l}\text { Indicador de desempenho dos vendedores em treinamentos de 'padrões de entrega dos } \\
\text { veículos', por concessionária - (média das notas dos cursos sobre procedimentos padrões por } \\
\text { concessionária + avaliação do cliente } 7 \mathrm{QS}) / 2 \text {. }\end{array}$ \\
\hline
\end{tabular}

Fonte: elaboração dos autores.

O Quadro 2 mostra os indicadores ou variáveis observadas, identificados por códigos e as suas respectivas definições, bem como o tipo de relação com cada variável latente, presente na Figura 1 Modelo Conceitual Proposto. Cabe ressaltar que o referido quadro foi desenvolvido levando em consideração a adoção da modelagem de equações estruturais na pesquisa, mas não foi evidenciado o método de análise adotado pela montadora.
Conforme constatado, mediante entrevistas não estruturadas com os gestores da área responsável, quanto ao método de análise adotado pela montadora, não houve evidências do agrupamento dos indicadores em variáveis latentes com a adoção de procedimentos estatísticos avançados. No entanto, constatou-se que os construtos SC, QS e TR eram intensos, presentes, priorizados, discutidos e inferidos de maneira intuitiva, fruto da experiência e da dedicação coletiva. Em relação aos indicadores 1TR e 2TR, 
diferentemente dos demais, merecem destaque e são particularizados no próximo tópico.

\subsection{Indicadores de desempenho em treinamento}

Foram adotadas, como critério para formação de indicadores de desempenho em treinamento (TR) das concessionárias, as médias das notas obtidas pelos vendedores para os cursos de atendimento nas vendas e dos cursos sobre padrões estabelecidos pela montadora para liberação dos veículos aos clientes. Foram também considerados na análise os indicadores 2QS e 7QS, que avaliam, respectivamente, $\mathrm{o}$ atendimento dos vendedores e o processo de liberação dos veículos aos clientes.

As médias das notas foram agrupadas por concessionária e relacionadas, por intermédio do $M S$ Access, aos indicadores 2QS e 7QS, também agrupadas por concessionária. Dessa forma, foi possível comparar o TR de cada concessionária para os cursos de atendimento nas vendas e dos padrões de entrega, com a respectiva avaliação do cliente acerca do atendimento do vendedor e o processo de liberação do veículo que vivenciou.
Constatou-se que o resultado da média aritmética simples entre TR no atendimento em vendas e a SC acerca do atendimento dos vendedores, pode ser interpretado como indicador do desempenho dos vendedores em treinamentos de atendimento, considerando o aproveitamento nos cursos e a avaliação dos clientes. O mesmo tratamento foi dado ao desempenho dos vendedores em treinamentos dos padrões de entrega e a avaliação do cliente acerca do processo de liberação do veículo.

Os resultados deram origem a dois indicadores: a) 1TR: Indicador de desempenho dos vendedores em treinamentos de atendimento nas vendas, por concessionária - (Média das notas dos cursos de atendimento por concessionária + avaliação do cliente 2QS)/2; e b) 2TR: Indicador de desempenho dos vendedores em treinamentos dos padrões de entrega dos veículos - (Média das notas dos cursos sobre procedimentos padrão por concessionária + avaliação do cliente 7QS)/2.

A formação dos indicadores 1TR e 2TR contribuiu para a construção do modelo e viabilizou a aplicação da modelagem de equações estruturais MEE, como indicadores formativos da variável latente TR. Cabe ressaltar que este estudo parte do entendimento de que o 
treinamento dos vendedores (TR) é uma variável latente dependente de variáveis independentes ou indicadores para sua estimação, ou seja, sua variação somente pode ser explicada a partir de seus indicadores. Conforme explicam Coltman et al. (2007), esse tipo de relação assume uma característica do tipo formativa, em que os indicadores ou variáveis observadas são a causa do construto.

\subsection{Amostragem}

Para o cálculo e análise do tamanho da amostra, foi utilizado o software G*Power 3.1.9 (FAUL et al., 2007 apud RINGLE; SILVA; BIDO, 2014). Conforme sugerem Faul et al. (2007, apud RINGLE; SILVA; BIDO， 2014)， os parâmetros utilizados para o cálculo por intermédio do $\mathrm{G}^{*}$ Power 3.1 .9 incluem: a definição do poder do teste, o tamanho do efeito e o número de preditores.

Os parâmetros utilizados para o cálculo do tamanho da amostra foram: poder (1- $\beta$ err prob) de 0,80 e tamanho do efeito $\left(\mathrm{f}^{2}\right)$ de 0,15 , conforme sugerido por Cohen (1998, apud RINGLE; SILVA; BIDO, 2014). A definição do número de preditores é realizada, conforme explicam os autores, pela observação do maior número de setas que chegam numa determinada variável latente.
Assim, foram observados, por intermédio da Figura 1, dois preditores, um partindo da variável latente QS e outro partindo da variável latente TR; ambos em direção à variável latente SC. Partindo-se dos parâmetros mencionados, bem como considerando o critério do dobro do resultado, conforme sugerido por Ringle, Silva e Bido (2014), seria necessária uma amostra de 136 concessionárias, 136 respondentes e 136 vendedores.

Os resultados se mostraram compatíveis com as quantidades constatadas a partir da análise documental dos dados secundários, quais sejam: 130 concessionárias, com uma diferença de seis concessionárias, se comparada com a quantidade ideal de 136; 936 respondentes à pesquisa de satisfação e 1.158 vendedores.

Constatada a representatividade das amostras, iniciou-se o tratamento estatístico dos dados, a partir das amostras de respondentes à pesquisa de satisfação da montadora e dos vendedores por concessionária.

\section{DESCRIÇÃO E ANÁLISE DOS RESULTADOS}

A análise dos dados foi realizada a partir dos resultados gerados pelos softwares Smart PLS 2.0 (RINGLE; 
WENDE; WILL, 2005) e o IBM SPSS Statistics 20. O método estatístico adotado neste estudo para a obtenção de respostas ao problema proposto foi a modelagem de equações estruturais - MEE, pelo método de estimação dos mínimos quadrados parciais - PLS-PM.

Conforme sugerido por Wong (2013) e Ringle, Silva e Bido (2014), a partir dos dados da amostra e a alocação das variáveis observadas nas suas respectivas variáveis latentes, foi processado o modelo no Smart PLS 2.0, cuja análise inicial focou os valores das cargas fatoriais, validade convergente, confiabilidade e validade discriminante dos modelos de mensuração reflexivos.

Constatou-se, inicialmente, que a carga da variável observada reflexiva '6QS' - test drive - do construto QS era de 0,463 , contra um valor que deve se aproximar de 0,70 (WONG, 2013). O que levou à exclusão desta variável e um ajuste do modelo. A eliminação desta variável pode ser explicada pela sua baixa aderência com a variável latente QS, ou seja, a percepção do cliente acerca da qualidade não atribui como essencial a oferta do test drive; que, na maioria das vezes, não é oferecido por questões ligadas à disponibilidade de veículos para este fim.

Constatadas que as cargas fatoriais estavam de acordo com o parâmetro, ou seja, valores aproximados ou maiores do que 0,70 foram adotados os testes de validade convergente, com a análise da variância média extraída ou AVE, e confiabilidade, com a análise da consistência interna e confiabilidade composta. Os resultados atenderam aos requisitos de obtenção de valores superiores a 0,50 para AVE (LOPES; PEREIRA; VIEIRA， 2009), valores superiores a 0,70 para a consistência interna e a confiabilidade composta (CHIN, 1996; HAIR Jr. et al., 2005). A Tabela 1 mostra os resultados mencionados com o ajuste do modelo e, adicionalmente, a raiz quadrada de AVE para a análise da validade discriminante:

Tabela 1 - Validade convergente e confiabilidade dos modelos reflexivos de mensuração

\begin{tabular}{ccccc}
\hline $\begin{array}{c}\text { Variáveis } \\
\text { Latentes }\end{array}$ & $\begin{array}{c}\text { AVE } \\
\text { Validade Convergente }\end{array}$ & $\begin{array}{c}\text { Confiabilidade } \\
\text { Composta }\end{array}$ & $\begin{array}{c}\alpha \text { de Cronbach } \\
\text { (Consistência } \\
\text { Interna) }\end{array}$ & $\sqrt{\boldsymbol{A V E}}$ \\
\hline QS & 0,54296 & 0,92853 & 0,91496 & 0,73686 \\
SC & 0,74654 & 0,92159 & 0,88611 & 0,86402 \\
\hline Parâmetros & AVE > 0,50 & Valores > 0,70 & Valores > 0,70 & \\
\hline \multicolumn{5}{r}{ Fonte: elaboração dos autores. }
\end{tabular}


Conforme evidencia a Tabela 1 , os resultados iniciais foram satisfatórios, considerando o ajuste no modelo que eliminou a variável observada ' $6 \mathrm{QS}$ ' - test drive. No entanto, ao se testar a validade discriminante pelo critério de Fornell e Lacker (1981), constatou-se que a raiz quadrada da AVE para a variável latente reflexiva QS, era menor que a sua correlação com a variável latente da SC:

Tabela 2 - Critério de Fornell e Lacker (1981)

para constatação de validade discriminante

\begin{tabular}{ccc}
\hline Variável Latente & QS & SC \\
\hline QS & $\mathbf{0 , 7 3 6 8 6}$ & $\mathbf{0 , 8 6 4 0 2}$ \\
SC & 0,82037 & Fonte: elaboração dos autores.
\end{tabular}

A Tabela 2 mostra o resultado do teste de validade discriminante que indicou a necessidade de reajuste no modelo, com a exclusão de novas variáveis. Conforme explicam Ringle, Silva e Bido (2014, p. 67), "devem-se retirar aquelas variáveis (uma a uma) que têm menores diferenças nas cargas fatoriais cruzadas", ou seja, os indicadores que apresentarem maiores correlações com as variáveis latentes. Nesse sentido, foram eliminadas no total, cinco variáveis observadas dos modelos de mensuração, quais sejam:

a) $2 \mathrm{SC}$ - satisfação do cliente com o atendimento;

b) $3 \mathrm{SC}$ - satisfação com os procedimentos adotados pela concessionária na entrega do veículo;

c) $2 \mathrm{QS}$ - avaliação do cliente acerca do atendimento prestado pelo vendedor; d) 6QS - avaliação do cliente acerca da oferta do vendedor para o teste drive; e

e) 7QS - avaliação do cliente acerca dos procedimentos adotados pela concessionária na liberação do veículo.

A eliminação das variáveis observadas: 2SC, 3SC, 2QS e 7QS fez sentido, pois além de terem apresentado as maiores cargas fatoriais, valores superiores à 0,9 , ao questionar o cliente sobre a sua satisfação geral - 1SC, deve-se considerar uma resposta que envolva o contexto de atendimento, entrega do veículo e procedimentos adotados na entrega.

Daí a justificativa da QS ter apresentado uma forte correlação de 0,82, com a SC. Em outras palavras, não haveria necessidade de avaliar-se a SC acerca do atendimento e a entrega de forma individualizada, já que o item 1SC 
abordava o conjunto destes atributos. Eliminadas as variáveis impeditivas da constatação de validade discriminante, o modelo foi novamente reprocessado no Smart PLS 2.0, o que resultou no modelo de mensuração, presente na Figura 2:

Figura 2 - Modelo de mensuração

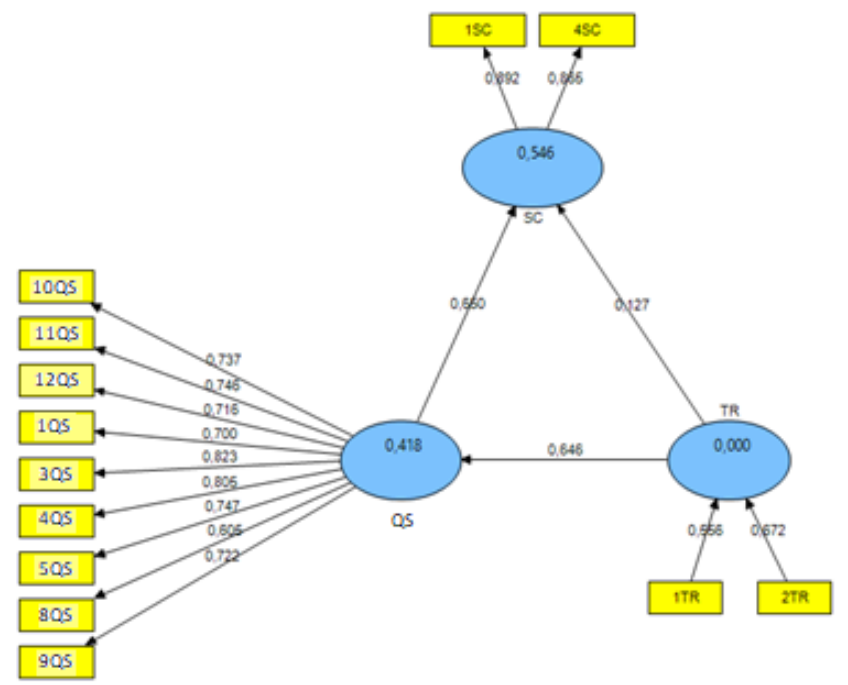

Fonte: elaborada pelos autores utilizando o Smart PLS 2.0

A Figura 2 apresenta o modelo ajustado, no qual estão presentes as variáveis latentes QS e SC e seus respectivos indicadores reflexivos, bem como a variável latente TR e seus respectivos indicadores formativos. No tocante, a variável latente reflexiva SC, composta por dois indicadores: 1SC e 4SC, segundo explicam Hair Jr. et al. (2005, p. 480) "um construto pode ser representado por dois indicadores, mas três é o número mínimo preferido de indicadores”.

A afirmação de Hair Jr. et al. (2005) é válida para variáveis latentes que de fato necessitem, mais do que dois indicadores para refleti-los, mas pode se mostrar inconclusiva para variáveis latentes em que um ou dois indicadores são suficientes para espelhá-las, ou que carreguem informações de múltiplos indicadores (BERGVIST; ROSSITER, 2007). Conforme explicam Bergkvist e Rossiter (2007, p. 183), "testes teóricos e achados empíricos permanecem inalterados, se substituídas as medidas de um único item, pelas medidas comumente utilizadas com múltiplos itens”.

A pesquisa de Drolet e Morrison (2001, p. 197) mostra que "mesmo com termos de erro muito modestos nas correlações entre os itens, a informação incremental a partir de cada item adicional é extremamente pequena". Os autores explicam ainda, que a inclusão desnecessária de indicadores sinônimos, ou seja, diferentes na designação e iguais em 
significado, podem superestimar a variável latente e pôr em risco a confiabilidade dos resultados.

Os pressupostos apontados por Drolet e Morrison (2001), Bergkvist e Rossiter (2007), reforçam a manutenção de apenas dois indicadores para a variável latente SC e a exclusão dos indicadores: 2SC, 3SC, 2QS e 7QS. Cabe destacar que tanto as recomendações de Hair Jr (2005), quanto Drolet e Morrison (2001) e Bergkvist e Rossiter (2007), são válidas para indicadores do tipo reflexivo, pois devem partilhar correlações para estimar a variável latente, não observada.

Quanto às análises de validade convergente pela variância média extraída (AVE), confiabilidade composta, consistência interna e validade discriminante pelo critério de Fornell e Lacker (1981), foram recalculadas com base no modelo ajustado (WONG, 2013; RINGLE; SILVA; BIDO, 2014). Os testes iniciais foram baseados em correlações e não contemplaram a análise do modelo de mensuração formativo (TR) que pressupõe a baixa ou a ausência de multicolineariedade entre as variáveis observadas ou indicadores (BIDO et al., 2010).

Nesse sentido, a Tabela 3 apresenta os resultados dos testes com o modelo ajustado para constatação da validade convergente e confiabilidade das variáveis latentes: QS e SC. Adicionalmente, foram dispostos os parâmetros referenciais para a análise dos testes, bem como as raízes quadradas de AVE utilizadas para constatação da validade discriminante:

Tabela 3 - Validade convergente e confiabilidade dos modelos reflexivos de mensuração ajustados

\begin{tabular}{rcccc}
\hline $\begin{array}{c}\text { Variáveis } \\
\text { Latentes }\end{array}$ & $\begin{array}{c}\text { AVE Validade } \\
\text { Convergente }\end{array}$ & $\begin{array}{c}\text { Confiabilidade } \\
\text { Composta }\end{array}$ & $\begin{array}{c}\alpha \text { de Cronbach } \\
\text { (Consistência } \\
\text { Interna) }\end{array}$ & $\sqrt{A V E}$ \\
\hline QS & 0,54143 & 0,91348 & 0,89275 & 0,73582 \\
$\mathrm{SC}$ & 0,77160 & 0,87105 & 0,70473 & 0,87841 \\
\hline Parâmetros & AVE > 0,50 & Valores $>0,70$ & Valores $>0,70$ & \\
\hline \multicolumn{5}{c}{ Fonte: elaboração dos autores. }
\end{tabular}

A Tabela 3 evidencia que os resultados dos testes para as variáveis latentes QS e SC, com o modelo ajustado foram compatíveis com os parâmetros sugeridos por Chin (1996), Hair Jr. et al.
(2005), Lopes, Pereira e Vieira (2009), respectivamente para: validade convergente - AVE $>0,50$; confiabilidade composta - Valores $>0,70$; e $\alpha$ de Cronbach - Valores $>0,70$. O resultado da 
validade discriminante, também, se modelo:

mostrou satisfatório após o ajuste do

Tabela 4 - Validade discriminante dos modelos reflexivos de mensuração ajustados

\begin{tabular}{ccc}
\hline $\begin{array}{c}\text { Variável } \\
\text { Latente }\end{array}$ & QS & SC \\
\hline QS & $\mathbf{0 , 7 3 5 8 2}$ & $\mathbf{0 , 8 7 8 4 1}$ \\
SC & 0,73235 & $\mathbf{c}$
\end{tabular}

A Tabela 4 evidencia que as raízes quadradas da variância média extraída (AVE) das variáveis latentes QS e SC são maiores que as suas correlações, o que permitiu constatar a validade discriminante no modelo ajustado. De acordo com Ringle, Silva e Bido (2014), a validade discriminante pode ser entendida como a constatação de que as cargas fatoriais de cada variável observada se agrupam em seus respectivos fatores ou variáveis latentes.

$$
\text { Constatadas as validades }
$$
convergente e discriminante, bem como a confiabilidade dos modelos de mensuração reflexivos foi iniciada a análise do modelo de mensuração formativo por intermédio do variance inflation factor (VIF) ou fator de inflação da variância. De acordo com Bido et al. (2010), o VIF é indicado para análise de modelos de mensuração formativos, a qual pressupõe a ausência de colineariedade ou multicolineariedade entre as variáveis observadas ou indicadores formativos das variáveis latentes.

Para a análise do VIF, conforme sugerido por Bido et al. (2010), foi adotada a regressão linear múltipla, por intermédio do software SPSS 20, que possui o teste para o diagnóstico de colineariedade e deve apresentar valores menores do que 0,50. O Quadro 3 evidencia os resultados da regressão linear múltipla: 
Quadro 3 - Resultado SPSS - Regressão linear múltipla para diagnóstico de multicolineariedade

\begin{tabular}{|c|c|c|c|c|c|c|c|c|}
\hline \multicolumn{9}{|c|}{ Coefficients $^{\mathrm{a}}$} \\
\hline \multirow[b]{2}{*}{ Model } & & \multicolumn{2}{|c|}{ Unstandardized Coefficients } & \multirow{2}{*}{\begin{tabular}{|c|}
$\begin{array}{c}\text { Standardized } \\
\text { Coefficients }\end{array}$ \\
Beta \\
\end{tabular}} & \multirow[b]{2}{*}{$t$} & \multirow[b]{2}{*}{ Sig. } & \multicolumn{2}{|c|}{ Collinearity Statistics } \\
\hline & & B & Std. Error & & & & Tolerance & VIF \\
\hline 1 & (Constant) & 1,376 &, 326 & & 4,224 & 000 & & \\
\hline & $1 T R$ &, 004 & ,004 & ,073 & 974 & ,331 & 897 & 1,114 \\
\hline & 2TR &,- 002 &, 004 &,- 042 &,- 553 &, 581 & 897 & 1,114 \\
\hline
\end{tabular}

a. Dependent Variable: VD

Fonte: elaborado pelos autores, via SPSS.

Foi admitida uma variável categórica qualquer para ser utilizada como variável dependente (VD) e os indicadores de desempenho dos vendedores nos treinamentos de atendimento e padrões de vendas (1TR e 2TR), como variáveis independentes, por intermédio do programa SPSS. Os resultados dos fatores de inflação da variância (VIF) extraídos da regressão mostraram valores de 1,114 , ou seja, superior a 0,50 , o que confirmou a presença fraca de multicolineariedade entre os indicadores 1TR e 2TR.
Não obstante, foram calculados por intermédio do módulo Bootstrapping do SmartPLS 2.0 os valores dos testes t, considerando 199 concessionárias (cases) e a reamostragem (samples) de 500, para: a) as cargas dos indicadores reflexivos; b) os coeficientes de caminhos do modelo estrutural; e c) os pesos dos indicadores formativos, para constatação da significância das relações (RINGLE; SILVA; BIDO, 2014). A Figura 3, a seguir, evidencia os valores dos testes t em cada relação, conforme sugerido por Ringle, Silva e Bido (2014): 
Figura 3 - Análise dos valores dos testes t

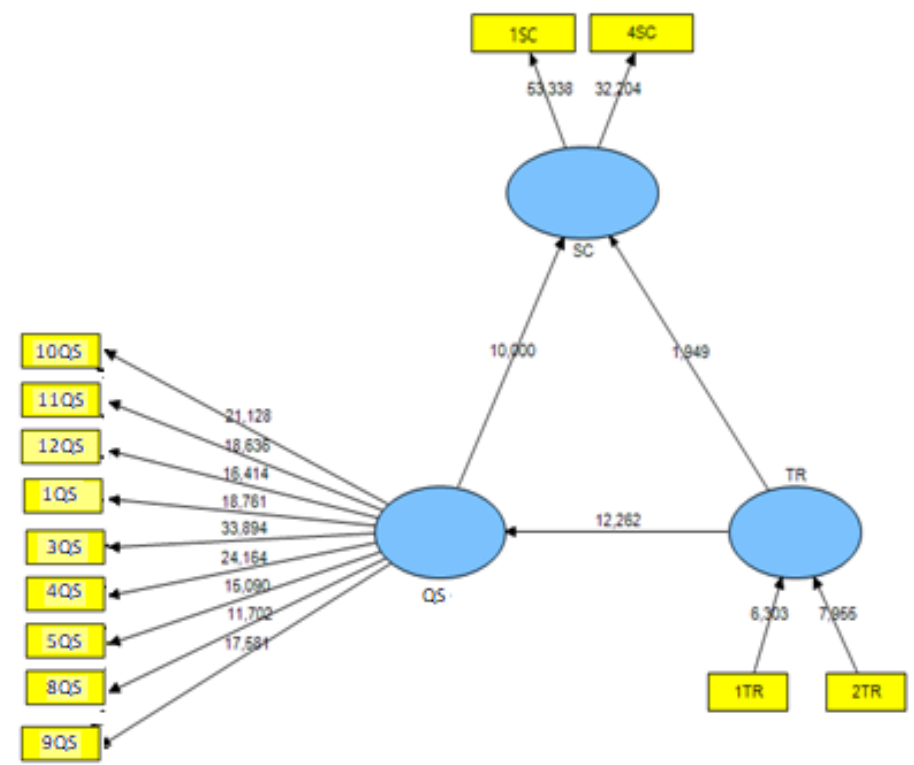

Fonte: elaborada pelos autores usando o SmartPLS.

A Figura 3 mostra os valores dos testes $\mathrm{t}$ para as cargas fatoriais, pesos e coeficientes de caminhos, em cada relação presente no modelo, quais sejam: a) entre as variáveis latentes QS, SC e TR modelo estrutural; b) variáveis latentes (QS e SC) e observáveis (1QS, 3QS, 4QS, 5QS, 8QS, 9QS, 10QS, 11QS e 12QS) - modelo reflexivo; e c) entre variáveis observáveis (1TR e 2TR) e variável latente (TR) modelo formativo.

Para Wong (2013), Ringle, Silva e Bido (2014), os valores dos testes t devem ser superiores a 1,96, indicando um nível de significância menor que 0,05 (p-valor< $0,05)$, a qual se rejeita a hipótese nula (H0) quando não houver relação significativa e se aceita a hipótese alternativa (H1) quando houver relação significativa.

Constatou-se por intermédio da Figura 3 que as relações entre: TR e QS, bem como entre QS e SC foram significativas, apresentando valores $\mathrm{t}$ superiores a 1,96, o que levou a aceitação das hipóteses H1 e H3. No entanto a relação entre TR e SC apresentou o valor-t de 1,95 , o que levou à rejeição da hipótese H2. A constatação de significância na relação entre QS e SC, bem como na relação entre TR e QS, implica em considerar que TR influencia direta e positivamente QS que, por sua vez, influencia SC. No entanto, observou-se que não há relação significativa entre TR e a 
SC, ou seja, TR não influencia diretamente a SC.

Após a análise das relações entre as variáveis latentes estimadas, foram analisados os coeficientes de determinação de Pearson $\left(\mathrm{R}^{2}\right)$ que, conforme Ringle, Silva e Bido (2014, p. 67), "avaliam a porção da variância das variáveis endógenas, que é explicada pelo modelo estrutural", bem como indicam a qualidade do modelo ajustado. Estes autores admitem como valores referenciais para análise do $\mathrm{R}^{2}$ os parâmetros sugeridos por Cohen (1988 apud RINGLE; SILVA; BIDO, 2014), quais sejam: $2 \%$ efeito pequeno, $13 \%$ efeito médio e $26 \%$ efeito grande.

Nesse sentido, constatou-se que $54,58 \%$ da variância na variável latente $\mathrm{SC}$, pode ser explicada pelas variáveis latentes QS e TR; bem como 41,77\% da variância na QS é explicada por TR. Ambas as variáveis latentes endógenas: QS e SC admitem um poder de explicação elevado, considerando um efeito superior a $26 \%$.

Conforme sugerido por Ringle, Silva e Bido (2014), buscou-se avaliar o poder de predição do modelo e a utilidade de cada variável latente, por intermédio dos indicadores de validade preditiva $\left(\mathrm{Q}^{2}\right)$ e do tamanho do efeito $\left(\mathrm{f}^{2}\right)$, obtidos pelo módulo Blindfolding do SmartPls. Os resultados mostraram que o modelo apresentou propriedade preditiva ao processar valores $\mathrm{Q}^{2}$ maiores do que 0 ( $\mathrm{QS}=0,21 ; \quad \mathrm{SC}=0,41$ e $\mathrm{TR}=0,06), \quad$ bem como apresentou valores $\mathrm{f}^{2} \quad(\mathrm{QS}=0,42$; $\mathrm{SC}=0,295$ e $\mathrm{TR}=0,06)$ entre os sugeridos por Cohen (1988 apud HENSELER et al., 2009), quais sejam: $0,02=$ pequeno; $0,15=$ médio e $0.35=$ grande.

\section{CONSIDERAÇÕES FINAIS}

Os resultados desta pesquisa confirmaram o pressuposto de que a qualidade percebida em serviços pelo cliente é uma determinante da sua satisfação. No entanto, observou-se que, diferentemente do que sugere a maior parte da revisão da literatura, não parece haver relação direta e significativa entre o treinamento dos vendedores das concessionárias e a satisfação dos clientes. A análise das relações entre as variáveis latentes SC, QS e TR, obtidas a partir dos testes t, revelaram a significância estatística de cada relação, o que levou à aceitação das hipóteses:

H1. A qualidade percebida pelos clientes, acerca dos serviços prestados pelos vendedores das concessionárias, influencia direta e positivamente a sua satisfação; e

H3. Os treinamentos realizados pelos vendedores das concessionárias 
influenciam direta e positivamente a qualidade percebida pelos clientes, acerca dos serviços prestados.

No entanto, a baixa significância estatística presente na relação entre TR e SC, levou à rejeição da hipótese:

$\mathrm{H} 2$. Os treinamentos realizados pelos vendedores das concessionárias influenciam direta e positivamente a satisfação dos clientes.

Os testes esclareceram a relação entre a variável latente TR e as variáveis latentes QS e SC: relação direta e positiva com QS na ordem de 0,646; relação muito fraca com SC na ordem de 0,127. Os coeficientes de determinação $\left(\mathrm{R}^{2}\right)$ das variáveis latentes $\mathrm{SC}$ e $\mathrm{QS}$, presentes no modelo estrutural, demonstraram que:

a) $54,58 \%$ da variância na satisfação dos clientes (SC) pode ser explicada pelas suas percepções acerca da qualidade em serviços (QS), considerando a ausência de significância entre TR e SC; e

b) $41,8 \%$ da variância na qualidade percebida em serviços (QS) pode ser explicada pelo treinamento dos vendedores (TR).

Em ambas as observações, os coeficientes denotam haver potencial de exploração de outras variáveis no modelo proposto ou em futuros estudos.
Face aos resultados apresentados, esta pesquisa respondeu ao problema a que se propôs, evidenciando que a satisfação dos clientes (SC) de uma rede de concessionárias de automóveis do estado do Rio Janeiro não parece ser influenciada diretamente pelo treinamento de vendedores (TR) da rede de concessionárias.

Em vez disso, o construto da qualidade percebida em serviços (QS) emerge para intermediar o efeito indireto do treinamento de vendedores (TR) na satisfação dos clientes (SC). Isso se deve, possivelmente, à própria natureza intangível da área de serviços, que exige, de antemão, o gerenciamento da impressão dos clientes, de suas expectativas e de suas percepções, para que só então o cliente possa ter a sensação de preencher suas necessidades e de satisfazer-se com o serviço prestado.

Apesar de não ter sido constatada uma relação direta e significante entre TR e SC, é possível um efeito indireto na satisfação dos clientes (SC), cuja variação de uma unidade no treinamento de vendedores (TR) causaria um efeito indireto de 0,419 na satisfação dos clientes (SC). Tal constatação foi suportada pela relação existente entre TR e QS e entre QS e $\mathrm{SC}(\mathrm{TR}=>\mathrm{QS}=>\mathrm{SC})$, em que a variação de uma unidade em TR significaria uma 
variação de 0,646 na QS, e uma variação de 0,646 na QS significaria uma variação de 0,419 na SC $(0,646 * 0,65)$.

Nesse sentido, pode-se concluir que o treinamento de vendedores (TR), objeto do presente estudo, é um elemento capaz de contribuir para o planejamento estratégico, para a criação de valor ao cliente e, consequentemente, para a geração de vantagem competitiva, mesmo não possuindo relação direta com a satisfação dos clientes (SC) como se acreditava inicialmente. Mediante os resultados apresentados, pode-se afirmar que os treinamentos oferecidos pela montadora à sua rede de concessionárias e aos vendedores estão ligados diretamente às percepções dos clientes acerca da qualidade em serviços.

Quanto às limitações deste estudo, podem-se destacar: a) no tratamento estatístico de dados secundários, provenientes de instrumento de pesquisa da satisfação, não houve a possibilidade de realização de pré-testes ou ajustes; e b) os dados que originaram os escores para avaliação do desempenho em treinamento dos vendedores consideravam a média geral por concessionária e curso realizado, ou seja, não houve o tratamento individualizado por colaborador, pois os dados já traziam a média geral de aproveitamento por curso e concessionária.

O presente estudo corrobora o estudo de Hernández et al. (2011), cujos resultados também apontaram para uma relação direta entre TR e QS, em vez de uma relação direta entre TR e SC. O modelo adotado neste estudo contribui para a Administração na avaliação dos resultados provenientes dos treinamentos, além de sugerir um método preditivo baseado naquilo que as organizações esperam, qual seja: o desenvolvimento profissional dos colaboradores, a satisfação dos clientes e sua consequente lealdade. 


\section{REFERÊNCIAS}

ANDERSON, E. W.; FORNELL, C.; LEHMANN, D. R. Customer satisfaction, market share, and profitability: findings from Sweden. Journal of Marketing, v. 58, p. 53-66, 1994.

; RUST, R. T. Customer satisfaction, productivity and profitability: differences between goods and services. Marketing Science, v. 16, n. 2, p. 129-145, 1997.

ANDERSON, E.W.; SULLIVAN, M.W. The antecedents and consequences of customer satisfaction for firms. Marketing Science, v. 12, n. 2, p. 125-143, 1993.

ANDREASSEN, T.W.; LINDESTAD, B. Customer loyalty and complex services: the impact of corporate image on quality, customer satisfaction and loyalty for customer with varying degrees of service expertise. International Journal of Service Industry Management, v. 9, n. 1, p. 7-23, 1998.

ANFAVEA - Associação Nacional dos Fabricantes de Veículos. Anuário, 2014-2017. Disponível em: < http://www.anfavea.com.br/estatisticas.html >. Acesso em 20 dez. 2017.

BARBOSA FILHO, F. H. A crise econômica de 2014/2017. Estudos Avançados, v. 31, n. 89, p. 51-60, 2017.

BENOSSI, G. Gestão do conhecimento no treinamento e desenvolvimento de pessoas. Dissertação (Mestrado em Ciência da Informação). Pontifícia Universidade Católica de Campinas, 2009.

BERGLVIST, L.; ROSSITER, J. R. The predictive validity of multiple-item versus singleitem measures of the same constructs. Journal of Marketing Research, v. 44, n. 2, p. 175184, 2007.

BIDO, D. S.; SILVA, D.; SOUZA, C. A.; GODOY, A. S. Mensuração de indicadores formativos nas pesquisas em administração de empresas: como lidar com a multicolineariedade entre eles? Administração: Ensino e Pesquisa, v. 11, n. 2, p. 245-269, 2010.

CHIN, W.W. Partial least squares is to Lisrel as principal components analysis is to common factor analysis. Technology Studies, v. 2, p. 315-319, 1996. 
COLTMAN, T.; DEVINNY, T. M.; MIDGLEY, D. F.; VENAIK, S. Formative versus reflective measurement models: Two applications of formative measurement. Journal of Business Research, v. 61, n. 12, p. 1250-1262, 2008.

COSTA, D. V. F.; CASTRO, D. R.; DUARTE, F. L.; TROCOLli, I. R.; ALTAF, J. G. Diagnóstico da necessidade de treinamento: o caso Beta Engenharia Ltda. Revista de Estudos do CEPE, n. 37, p. 190-214, 2013.

CRONIN, J.; TAYLOR, S. Measuring service quality: a reexamination and extension. Journal of Marketing, v. 56, p. 55-68, 1992.

CRONIN JR, J. J.; BRADY, M. K.; HULT, G. T. M. Assessing the effects of quality, value, and customer satisfaction on consumer behavioral intentions in service environments. Journal of Retailing, v. 76, n. 2, p. 193-218, 2000.

DENTON, D. K. Qualidade em serviços: o atendimento ao cliente como fator de vantagem competitiva. São Paulo: McGraw-Hill, 1990.

DROLET, A. L.; MORRISON, D. G. Do we really need multiple-item measures in service research? Journal of Service Research, v. 3, n. 3, p. 196-204, 2001.

ELLINGER, A.; SHIN, H.; NORTHINGTON, W. M.; ADAMS, F. G. The influence of supply chain management competency on customer satisfaction and shareholder value. Supply chain management: an international journal, v. 17, n. 3, p. 249-262, 2012.

FENABRAVE - Federação Nacional da Distribuição de Veículos Automotores. Índices e Números. $\quad$ Disponível em: <http://www3.fenabrave.org.br:8082/plus/modulos/listas/index.php?tac=indices-enumeros\&idtipo=6\&layout=indices-e-numeros $>$. Acesso em: 20 dez. 2017.

FORNELL C.; CHA J. Partial Least Squares. Advanced Methods of Marketing Research. Cambridge: MA, p. 52-78, 1994.

FORNELL, C.; JOHNSON, M.; ANDERSON, E. W.; CHA, J.; BRYANT, B. The American Customer Satisfaction Index: nature, purpose and findings. Journal of Marketing, v. 60, p. 7-18, 1996.

; LARCKER, D.F. Evaluating structural equation models with unobservable variables and measurement error. Journal of Marketing Research, v. 18, n. 1, p. 39-50, 1981. 
The satisfied customer: winners and losers in the battle for buyer preference. London: Palgrave Macmillan, 2007.

GIL, A. C. Como elaborar projetos de pesquisa. 4a. Ed. São Paulo: Atlas, 2002.

HAIR Jr., J.F.; SARSTEDT, M.; RINGLE, C.M.; MENA, J.A. An assessment of the use of partial least squares structural equation modeling in marketing research. Journal of the Academy of Marketing Science, v. 40, n. 3, p. 414-433, 2012.

HAIR Jr., J. F.; ANDERSON, R. E.; TATHAM, R. L.; BLACK, W. C. Análise multivariada de dados. 5a Ed. Porto Alegre: Bookman, 2005.

HENSELER, J.; RINGLE, C. M.; SINKOVICS, R. R. The use of partial least squares path modeling in international marketing. Advances in International Marketing, v. 20, p. 277319, 2009.

HERnÁNDEZ, R. M. S.; CASTRO, B. A. A.; JUÁREZ, J. R.; RIVAS, S. A. H. La capacitación como predictora de satisfacción del cliente em organizaciones turísticas. Ingeniería Industrial, v. 32, n. 3, p. 207-212, 2011.

LIEN, C. H.; CAO, Y.; ZHOU, X. Service quality, satisfaction, stickiness, and usage intentions: an exploratory evaluation in the context of WeChat services. Computers in Human Behavior, v. 68, p. 403-410, 2017.

LOPES, H. E. G.; PEREIRA, C. C. P.; VIEIRA, A. F. S. Comparação entre os modelos Norte-Americano (ACSI) e Europeu (ECSI) de satisfação do cliente: um estudo no setor de serviços. Revista de Administração Mackenzie, v. 10, n. 1, p. 161-186, 2009.

MALHOTRA, N. K. Pesquisa de marketing: uma orientação aplicada. 4a Ed. Porto Alegre: Bookman, 2006.

MOURA, A. C.; GONÇALVES, C. A. Modelo de satisfação ACSI modificado no setor de telefonia móvel. Revista de Administração de Empresas, Edição especial,v.45, p. 72-85, 2005.

OLIVER, R. L. A Cognitive Model of the Antecedents and Consequences of Satisfaction Decisions. Journal of Marketing Research, v. 17, n. 4, p. 460-469, 1980. 
PARASURAMAN, A.; ZEITHAML, V. A.; BERRY, L. L. Servqual: a multiple-item scale for measuring consumer perceptions of service quality. Journal of Retailing, v. 64, n. 1, p. 12-340, 1988.

RAHMAN, M. S.; KHAN, A. H.; HAQUE, M. M. A conceptual study on the relationship between service quality towards customer satisfaction: SERQUAL and Gronroos's Service Quality Perspective. Asian Social Science, v. 8, n. 13, p. 201-210, 2012.

RAPHAËLE, M.; RAFER, D. Training and power in franchise systems: the example of the UK automotive industry. Journal of European Industrial Training, v. 22, n. 9, p. 367-374, 1998.

REYNOLD, J; JONES, R. Transferring the Toyota lean cultural paradigm into India: implications for human resource management. The International Journal of Human Resource Management, v. 25, n. 15, p. 2174-2191, 2014.

RINGLE, C. M.; SILVA, D; BIDO, D. Modelagem de equações estruturais com utilização do SmartPLS. Revista Brasileira de Marketing - Ed. Especial, v. 13, n. 2, p. 54-71, 2014.

RINGLE, C.M.; WENDE, S.; WILL, S. SmartPLS 2.0 (M3) Beta. Hamburg 2005. Disponível em: <http://www.smartpls.de.> Acesso em: 15 jul. 2014.

ROCKENBACH JÚNIOR, P. C.; COELHO, K. S.; DELLAGNELO, E. H. L.; KANITZ, A. F. Reflexões sobre uma perspectiva crítica na concepção do processo de capacitação dos empregados da escola Alpha. Revista Gestão e Planejamento, v. 13, n. 1, p. 177-198, 2013.

ROS, S. C.; BENAVENT, F.B. A gestão de qualidade no setor de serviços na Espanha: enfoques, motivos e expectativas. Revista de Administração - RAUSP, v. 37, n. 4, p. 6-15, 2002 ,

SALAS, E.; CANNON-BOWERS, J.A. The science of training: a decade of progress. Annual Review Psychology, v. 52, p. 471-499, 2001.

SILVA, E. M. Mensuração da satisfação e desempenho organizacional: um estudo em uma empresa de varejo. Dissertação (Mestrado em Administração) apresentada à Faculdade de Ciências Empresariais da Universidade FUMEC, Belo Horizonte, 2006.

SILVA, M. F. O.; BERTRAND, H. Supply Chain, Satisfação dos Clientes e o Custo dos Inventários: um modelo para otimização. RAC-Eletrônica, v. 2, n. 2, p. 218-233, 2008. 
SPARROW, P.; OTAYE-EBEDE, L. Lean management and HR function capability: the role of HR architecture and the location of intellectual capital. The International Journal of Human Resource Management, v. 25, n. 21, p. 2892-2910, 2014.

STAVRINOUDIS, T.; PSIMOULIS, M. How do education and training policies determine customer satisfaction and hotels' performance? European Journal of Tourism Research, v. 17, p. 177-190, 2017.

STURGEON, T.; BIESEBROECK, J.V.; GEREFFI, G. Value chains, networks and clusters: reframing the global automotive industry. Journal of Economic Geography, v. 8, n. 3, p. 297-321, 2008.

TABACHNICK, B. G.; FIDELL, L. S. Using multivariate statistics. 5th ed. New York: Harper Collins, 2007.

TASCA, J. E.; ENSSLIN, L.; ENSSLIN, S. R. A avaliação de programas de capacitação: um estudo de caso na administração pública. Revista de Administração Pública, v. 46, n. 3, p. 647-675, 2012.

THIRKELL, E.; ASHMAN, I. Lean towards learning: connecting Lean Thinking and human resource management in UK higher education. The International Journal of Human Resource Management, v. 25, n. 21, p. 2957-2977, 2014.

URDAN, A. T.; RODRIGUES, A. R. O modelo de índice de satisfação do cliente norteamericano: um exame inicial no Brasil com equações estruturais. Revista de Administração Contemporânea - RAC, v. 3, n. 3, p. 109-130, 1999.

VIEIRA, V.A.; MATOS, C.A.; SLONGO, L.A. Avaliação das relações entre qualidade de serviço do site, satisfação, valor percebido, lealdade e boca a boca por meio de um modelo teórico. Revista de Administração - RAUSP, v. 44, n. 2, p. 131-146, 2009.

WALIGÓRA, J.; WALIGÓRA, R. Measuring customer satisfaction and loyalty in the automotive industry: a case of premium brand of passenger cars. Dissertation (Master in Marketing and Statistics). Aarhus School of Business, 2007.

WILLIANS, P.; NAUMANN, E. Customer satisfaction and business performance: a firm level analysis. Journal of Services Marketing, v. 25, n. 1, p. 20-32, 2011.

WONG, K. K. K. Partial least squares structural equation modeling (PLS-SEM): techniques using SmartPLS. Marketing Bulletin, v. 24, n. 1, 2013. 